\title{
Existence of positive solutions for a semilinear Schrödinger equation in $\mathbb{R}^{N}$
}

Houqing Fang ${ }^{1,2^{*}}$ and Jun Wang ${ }^{2}$

\section{"Correspondence:}

doudou@ujs.edu.cn

'School of Economics and

Management, Southeast University,

Nanjing, 210096, China

${ }^{2}$ Faculty of Science, Jiangsu

University, Zhenjiang, Jiangsu

212013, P.R. China

\begin{abstract}
In this paper, we study the existence of multi-bump solutions for the semilinear Schrödinger equation $-\Delta u+(1+\lambda a(x)) u=(1-\lambda b(x))|u|^{p-2} u, \forall u \in H^{1}\left(\mathbb{R}^{N}\right)$, where $N \geq 1,2<p<2 N /(N-2)$ if $N \geq 3, p>2$ if $N=2$ or $N=1, a(x) \in C\left(\mathbb{R}^{N}\right)$ and $a(x)>0$, $b(x) \in C\left(\mathbb{R}^{N}\right)$ and $b(x)>0$. For any $n \in \mathbb{N}$, we prove that there exists $\lambda(n)>0$ such that, for $0<\lambda<\lambda(n)$, the equation has an $n$-bump positive solution. Moreover, the equation has more and more multi-bump positive solutions as $\lambda \rightarrow 0$.
\end{abstract}

MSC: $35 \mathrm{~J} 61 ; 35 \mathrm{~J} 20 ; 35 \mathrm{Q} 55 ; 49 J 40$

Keywords: multi-bump solution; semilinear Schrödinger equation; variational methods

\section{Introduction and main results}

In this paper we study the following time independent semilinear Schrödinger equation:

$$
\left(\mathcal{S}_{\lambda}\right) \quad-\Delta u+(1+\lambda a(x)) u=(1-\lambda b(x))|u|^{p-2} u, \quad \forall u \in H^{1}\left(\mathbb{R}^{N}\right)
$$

where $N \geq 1,2<p<2^{*}, 2^{*}$ is the critical Sobolev exponent defined by $2^{*}=\frac{2 N}{N-2}$ if $N \geq 3$ and $2^{*}=\infty$ if $N=2$ or $N=1$, and $\lambda>0$ is a parameter.

This kind of equation arises in many fields of physics. For the following nonlinear Schrödinger equation:

$$
i \hbar \frac{\partial \varphi}{\partial t}=-\frac{\hbar^{2}}{2 m} \Delta \varphi+N(x) \varphi-f(x,|\varphi|) \varphi,
$$

where $i$ is the imaginary unit, $\Delta$ is the Laplacian operator, and $\hbar>0$ is the Planck constant. A standing wave solution of (1.1) is a solution of the form

$$
\varphi(x, t)=u(x) e^{-\frac{i E t}{\hbar}}, \quad u(x) \in \mathbb{R}
$$

Thus, $\varphi(x, t)$ solves (1.1) if and only if $u(x)$ solves the equation

$$
-\hbar^{2} \Delta u+V(x) u=g(x, u)
$$


where $V(x)=N(x)-E$ and $g(x, u)=f(x,|u|) u$. The function $V$ is called the potential of (1.2). If $g(x, u)=(1-\lambda b(x))|u|^{p-2} u$, then (1.2) can be written as

$$
-\hbar^{2} \Delta u+V(x) u=(1-\lambda b(x))|u|^{p-2} u .
$$

If $\hbar=1$ and $V(x)=1+\lambda a(x)$, then (1.3) is reduced to $\left(\mathcal{S}_{\lambda}\right)$.

The nonlinear Schrödinger equation $\left(\mathcal{S}_{\lambda}\right)$ models some phenomena in physics, for example, in nonlinear optics, in plasma physics, and in condensed matter physics, and the nonlinear term simulates the interaction effect, called the Kerr effect in nonlinear optics, among a large number of particles; see, for example, [1,2]. The case of $p=4$ and $N=3$ is of particular physical interest, and in this case the equation is called the Gross-Pitaevskii equation; see [3].

The limiting equation of $\left(\mathcal{S}_{\lambda}\right)$ is

$$
-\Delta u+u=|u|^{p-2} u, \quad u \in H^{1}\left(\mathbb{R}^{N}\right),
$$

as $\lambda \rightarrow 0$. It is well known that (1.4) has a unique positive radial solution $z$, which decays exponentially at $\infty$. This $z$ will serve as a building block to construct multi-bump solutions of $\left(\mathcal{S}_{\lambda}\right)$. For $n \in \mathbb{N}$, let $y_{1}, \ldots, y_{n} \in \mathbb{R}^{N}$ be the sufficiently separated points. The profile of the function $\sum_{i=1}^{n} z\left(x-y_{i}\right)$ resembles $n$ bumps and accordingly a solution of $\left(\mathcal{S}_{\lambda}\right)$ which is close to $\sum_{i=1}^{n} z\left(x-y_{i}\right)$ in $H^{1}\left(\mathbb{R}^{N}\right)$ is called an $n$-bump solution.

As we know, multi-bump solutions arise as solutions of (1.2) as $\hbar \rightarrow 0$, under the assumption that $V$ has several critical points; see for example [4-7]. Particularly, in the interesting paper [5], the authors proved that the solutions of (1.2) have several peaks near the point of a maximum of $V$. These peaks converge to the maximum of $V$ as $\hbar \rightarrow 0$. Actually, there have been enormous studies on the solutions of (1.2) as $\hbar \rightarrow 0$, which exhibit a concentration phenomenon and are called semiclassical states. In the early results, most of the researchers focused on the case $\inf _{x \in \mathbb{R}^{N}} V(x)>0$ and $g$ is subcritical. Here and in the sequel, we say $g$ is subcritical if $g(x, u) \leq C|u|^{p-1}$ for $2 \leq p<2^{*}$ with $2^{*}:=2 N /(N-2)(N \geq 3)$, and $g$ is critical or supercritical if $c_{1}|u|^{2^{*}-1} \leq g(x, u) \leq c_{2}|u|^{2^{*}-1}$ or only $c_{1}|u|^{2^{*}-1} \leq g(x, u)$ for all large $|u|$. In the case of $\inf _{x \in \mathbb{R}^{N}} V(x)>0$, Floer and Weinstein in [8] first considered $N=1, g(u)=u^{3}$. Using the Lyapunov-Schmidt reduction argument, they proved that the system (1.2) has spike solutions, which concentrate near a nondegenerate critical point of the potential $V$. This result was extended to the high dimension case with $N \geq 2$ and for $g(u)=|u|^{p-2} u$ by Oh [7,9]. If the potential $V$ has a nondegenerate critical point, Rabinowitz [10] obtained the existence result for (1.2) with $\hbar$ small, provided that $0<\inf _{x \in \mathbb{R}^{N}} V(x)<\liminf _{|x| \rightarrow \infty} V(x)$. Using a global variational argument, Del Pino and Felmer [11, 12] established the existence of multi-peak solutions having exactly $k$ maximum points provided that there are $k$ disjoint open bounded sets $\Omega_{i}$ such that $\inf _{x \in \partial \Omega_{i}} V(x)>\inf _{x \in \Omega_{i}} V(x)$, each $\Omega_{i}$ having one peak concentrating at its bottom. For the subcritical case, Refs. $[1,6,13-15]$ also proved that the solutions of (1.2) are concentrated at critical points of $V$. There have also been recent results on the existence of solutions concentrating on manifolds; for instance, see [16-18] and the references therein.

If $g$ is subcritical, Refs. [19, 20] first obtained the semiclassical solutions of (1.2) with critical frequency, i.e., $\inf _{\in \mathbb{R}^{N}} V(x)=0$. They exhibited new concentration phenomena for bound states and their results were extended and generalized in [3, 21, 22]. Later, 
if $\inf _{\in \mathbb{R}^{N}} V(x)=0$, Ding and Lin [23] obtained semiclassical states of (1.2) when the nonlinearity $g$ is of the critical case. Recently, if the potentials $V$ change sign, that is, $\inf _{x \in \mathbb{R}^{N}} V(x)<0$, Refs. [24, 25] proved that the system (1.2) has semiclassical states.

Some researchers had also obtained multi-bump solutions for the equation

$$
-\Delta u+V(x) u=f(x, u), \quad u \in H^{1}\left(\mathbb{R}^{N}\right)
$$

where $V$ and $f$ are $T_{i}$ periodic in $x_{i}$. Coti Zelati and Rabinowitz [26] first constructed multi-bump solutions for the Schrödinger equation (1.5). The building blocks are onebump solutions at the mountain pass level and the existence of such solutions as well as multi-bump solutions is guaranteed by a nondegeneracy assumption of the solutions near the mountain pass level. Later, under the same nondegeneracy assumption, Coti Zelati and Rabinowitz in [27] constructed multi-bump solutions for periodic Hamiltonian systems. Multi-bump solutions have also been obtained for asymptotically periodic Schrödinger equations by Alama and Li [28]. For subsequent studies in this direction, for example, see [29-35] and the references therein. Recently, Refs. [36-38] also proved the existence of multi-bump solutions in other elliptic equations.

In this paper, we are interested in constructing multi-bump solutions of $\left(\mathcal{S}_{\lambda}\right)$ with $\lambda$ small enough. Similar results have been obtained in $[39,40]$ for the equations

$$
-\Delta u+(1+\lambda a(x)) u=|u|^{p-2} u, \quad u \in H^{1}\left(\mathbb{R}^{N}\right)
$$

and

$$
-\Delta u+u=(1-\lambda a(x))|u|^{p-2} u, \quad u \in H^{1}\left(\mathbb{R}^{N}\right) .
$$

To state the main result for $\left(\mathcal{S}_{\lambda}\right)$, we need the following conditions on the functions $a$ and $b$ :

$$
\begin{gathered}
\left(\mathcal{R}_{1}\right) a(x)>0 \text { and } a(x) \in C\left(\mathbb{R}^{N}\right), b(x)>0 \text { and } b(x) \in C\left(\mathbb{R}^{N}\right) \text {, and } \\
\lim _{|x| \rightarrow \infty} a(x)=\lim _{|x| \rightarrow \infty} b(x)=0 .
\end{gathered}
$$

$\left(\mathcal{R}_{2}\right)$ One of the following holds: (i) $\lim _{|x| \rightarrow \infty} \frac{\ln (a(x))}{|x|}=0$; (ii) $\lim _{|x| \rightarrow \infty} \frac{\ln (b(x))}{|x|}=0$.

Theorem 1.1 Suppose that the assumptions $\left(\mathcal{R}_{1}\right)$ and $\left(\mathcal{R}_{2}\right)$ hold. Then for any positive integer $n$ there exists $\lambda(n)>0$ such that, for $0<\lambda<\lambda(n)$, the system $\left(\mathcal{S}_{\lambda}\right)$ has an $n$-bump positive solution. As a consequence, for any positive integer $n$, there exists $\lambda_{1}(n)>0$ such that, for $0<\lambda<\lambda_{1}(n)$, the system $\left(\mathcal{S}_{\lambda}\right)$ has at least $n$ positive solutions.

Similar to [39, 40], the solutions in Theorem 1.1 do not concentrate near any point in the space. Instead, the bumps of the solutions we obtain are separated far apart and the distance between any pair of bumps goes to infinity as $\lambda \rightarrow 0$. The size of each bump does not shrink and is fixed as $\lambda \rightarrow 0$. This is in sharp contrast to the concentration phenomenon described above. This phenomenon has been observed by D'Aprile and Wei in [41] for a Maxwell-Schrödinger system. 
We shall use the variational reduction method to prove the main results. Our argument is partially inspired by [39-42]. This paper is organized as follows. In Section 2, preliminary results are revisited. We prove Theorem 1.1 in Section 3.

\section{Some preliminary works}

\subsection{Variational framework}

In this section, we shall establish a variational framework for the system $\left(\mathcal{S}_{\lambda}\right)$. For convenience of notation, let $C$ and $C_{i}$ denote various positive constants which may be variant even in the same line. In the Hilbert space $H^{1}\left(\mathbb{R}^{N}\right)$, we shall use the usual inner product,

$$
\langle u, v\rangle=\int_{\mathbb{R}^{N}} \nabla u \cdot \nabla v+u v,
$$

and the induced norm $\|u\|=\langle u, u\rangle^{\frac{1}{2}}$. Let $|\cdot|_{q}$ denote the usual $L^{q}\left(\mathbb{R}^{N}\right)$-norm and $(\cdot, \cdot)_{2}$ be the usual $L^{2}\left(\mathbb{R}^{N}\right)$-inner product. Let $n \in N$. We shall use $\sum_{i<j}$ and $\sum_{i \neq j}$ to represent summation over all subscripts $i$ and $j$ satisfying $1 \leq i<j \leq n$ and $1 \leq i \neq j \leq n$, respectively. Let us first introduce some basic inequalities which will be used later.

The following four lemmas are taken from $[39,40]$.

Lemma 2.1 For $q>1$, there exists $C>0$ such that, for any real numbers $a$ and $b$,

$$
|| a+\left.b\right|^{q}-|a|^{q}-\left.|b|^{q}|\leq C| a\right|^{q-1}|b|+C|b|^{q-1}|a| .
$$

Lemma 2.2 For $q \geq 2$, there exists $C>0$ such that, for any $a>0$ and $b \in \mathbb{R}$,

$$
|| a+\left.b\right|^{q}-a^{q}-q a^{q-1} b \mid \leq C\left(a^{q-2}|b|^{2}+|b|^{q}\right) .
$$

Lemma 2.3 For $q \geq 2, n \in N$, and $a_{i} \geq 0, i=1, \ldots, n$,

$$
\left(\sum_{i=1}^{n} a_{i}\right)^{q} \geq \sum_{i=1}^{n} a_{i}^{q}+(q-1) \sum_{i \neq j}^{n} a_{i}^{q-1} a_{j}
$$

and

$$
\left(\sum_{i=1}^{n} a_{i}\right)^{q} \geq \sum_{i=1}^{n} a_{i}^{q}+q \sum_{1 \leq i<j \leq n}^{n} a_{i}^{q-1} a_{j} .
$$

Lemma 2.4 For $q \geq 2$, there exists $C>0$ such that, for any $a_{i} \geq 0, i=1, \ldots, n$,

$$
\left[\left(\sum_{i=1}^{n} a_{i}\right)^{q-1}-\sum_{i=1}^{n} a_{i}^{q-1}\right]^{\frac{q}{q-1}} \leq C \sum_{i \neq j} a_{i}^{q-1} a_{j} .
$$

Recall that, for $2<p<2^{*}$, the unique positive solution of the equation

$$
-\Delta u+u=|u|^{p-2} u, \quad u \in H^{1}\left(\mathbb{R}^{N}\right)
$$

has the following properties; see, for example, [40, 43-45]. 
Lemma 2.5 If $2<p<2^{*}$, then every positive solution of (2.1) has the form $z_{y}:=z(\cdot-y)$ for some $y \in \mathbb{R}^{N}$, where $z \in C^{\infty}\left(\mathbb{R}^{N}\right)$ is the unique positive radial solution of (2.1) which satisfies, for some $c>0$,

$$
z(r) r^{\frac{N-1}{2}} e^{r} \rightarrow c>0, \quad z^{\prime}(r) r^{\frac{N-1}{2}} e^{r} \rightarrow-c>0, \quad \text { as } r=|x| \rightarrow \infty .
$$

Furthermore, if $\beta_{1} \leq \cdots \leq \beta_{n} \leq \cdots$ are the eigenvalues of the problem

$$
-\Delta v+v=\beta z^{p-2} v, \quad v \in H^{1}\left(\mathbb{R}^{N}\right),
$$

then $\beta_{1}=1, \beta_{2}=p-1$, and the eigenspaces corresponding to $\beta_{1}$ and $\beta_{2}$ are spanned by $z$ and $\left\{\partial z / \partial x_{\alpha} \mid \alpha=1, \ldots, N\right\}$, respectively.

We shall use $z_{y}$ as building blocks to construct multi-bump solutions of $\left(\mathcal{S}_{\lambda}\right)$. For $y_{i}, y_{j} \in$ $\mathbb{R}^{N}$, the identity

$$
\int_{\mathbb{R}^{N}} z_{y_{i}}^{p-1} z_{y_{j}}=\left\langle z_{y_{i}}, z_{y_{j}}\right\rangle=\int_{\mathbb{R}^{N}} z_{y_{i}} z_{y_{j}}^{p-1}
$$

will be frequently used in the sequel. The following lemma is a consequence of Lemma 2.4 in [46] (see also Lemma II.2 of [47]).

Lemma 2.6 There exists a positive constant c $>0$ such that, as $\left|y_{i}-y_{j}\right| \rightarrow \infty$,

$$
\int_{\mathbb{R}^{N}} z_{y_{i}}^{p-1} z_{y_{j}} \sim c\left|y_{i}-y_{j}\right|^{-\frac{N-1}{2}} e^{-\left|y_{i}-y_{j}\right|}
$$

For $h>0, n \geq 2$, and $n \in \mathbb{N}$, define

$$
\mathcal{D}_{h}=\left\{\left(y_{1}, \ldots, y_{n}\right) \in\left(\mathbb{R}^{N}\right)^{n}|| y_{i}-y_{j} \mid>h \text { for } i \neq j\right\} .
$$

For convenience, we make the convention

$$
\mathcal{D}_{h}=\mathbb{R}^{N}, \quad \text { if } n=1 .
$$

For $y=\left(y_{1}, \ldots, y_{n}\right) \in \mathcal{D}_{h}$, denote

$$
\begin{aligned}
& u_{y}(x)=\sum_{i=1}^{n} z\left(x-y_{i}\right), \\
& \mathcal{T}_{y}=\left\{\frac{\partial z\left(\cdot-y_{i}\right)}{\partial x_{\alpha}} \mid \alpha=1, \ldots, N, i=1, \ldots, n\right\}
\end{aligned}
$$

and

$$
\mathcal{W}_{y}=\left\{v \in H^{1}\left(\mathbb{R}^{N}\right) \mid\langle v, u\rangle=0, \forall u \in \mathcal{T}_{y}\right\} .
$$

Then $H^{1}\left(\mathbb{R}^{N}\right)=\mathcal{T}_{y} \oplus \mathcal{W}_{y}$. Set $P_{\lambda}(x)=1-\lambda b(x), V_{\lambda}(x)=1+\lambda a(x), \mathcal{N}_{\lambda}=(p-1)\left(-\Delta+V_{\lambda}\right)^{-1}$, and $\mathcal{N}_{0}=\mathcal{N}$. For $y \in \mathcal{D}_{h}$ and $\varphi \in H^{1}\left(\mathbb{R}^{N}\right)$, define

$$
\mathcal{K}_{y}=\varphi-\sum_{i=1}^{n} \mathcal{N}\left(z^{p-2}\left(\cdot-y_{i}\right) \varphi\right)+\sum_{i=1}^{n} L_{i} \varphi
$$


where

$$
\sum_{i=1}^{n} L_{i} \varphi=\sum_{i \neq j} \sum_{\alpha=1}^{N}\left\langle\mathcal{N}\left(z^{p-2}\left(\cdot-y_{j}\right) \varphi\right), \frac{\partial z\left(\cdot-y_{i}\right)}{\partial x_{\alpha}}\right\rangle\left\|\frac{\partial z\left(\cdot-y_{i}\right)}{\partial x_{\alpha}}\right\|^{-2} \frac{\partial z\left(\cdot-y_{i}\right)}{\partial x_{\alpha}} .
$$

Noting that $\mathcal{K}_{y} \mid \mathcal{W}_{y}: \mathcal{W}_{y} \rightarrow \mathcal{W}_{y}$ has the form identity-compact.

Lemma 2.7 (See Lemma 2.3 of [40]) If $h \rightarrow \infty$, then

$$
\left|u_{y}\right|^{p-2}-\sum_{i=1}^{n} z^{p-2}\left(\cdot-y_{i}\right) \rightarrow 0
$$

in $L^{p /(p-2)}\left(\mathbb{R}^{N}\right)$ uniformly in $y \in \mathcal{D}_{h}$.

Lemma 2.8 (See Lemma 2.4 of [40]) Let $u, v \in H^{1}\left(\mathbb{R}^{N}\right)$. If $v \rightarrow 0$, then

$$
|u+v|^{p-1}-|u|^{p-2} \rightarrow 0
$$

in $L^{p /(p-2)}\left(\mathbb{R}^{N}\right)$ uniformly in $u$ in any bounded set.

Lemma 2.9 (See Lemma 2.5 of [40]) There exist $h_{0}>0$ and $\eta_{0}>0$ such that, for $h>h_{0}$ and $y \in \mathcal{D}_{h}, \mathcal{K}_{y} \mid \mathcal{W}_{y}: \mathcal{W}_{y} \rightarrow \mathcal{W}_{y}$ is invertible and

$$
\left\|\left(\mathcal{K}_{y} \mid \mathcal{W}_{y}\right)^{-1}\right\| \leq \eta_{0}
$$

Lemma 2.10 Let $v \in H^{1}\left(\mathbb{R}^{N}\right)$. If $\lambda \rightarrow 0, v \rightarrow 0$, and $h \rightarrow \infty$, then

$$
\sup _{y \in \mathcal{D}_{h}, \varphi \in H^{1}\left(\mathbb{R}^{N}\right),\|\varphi\|=1}\left\|\mathcal{K}_{y} \varphi-\left(\varphi-\mathcal{N}_{\lambda}\left(P_{\lambda}\left|u_{y}+\nu\right|^{p-2} \varphi\right)\right)\right\| \rightarrow 0
$$

and

$$
\sup _{y \in \mathcal{D}_{h}, \varphi \in H^{1}\left(\mathbb{R}^{N}\right),\|\varphi\|=1}\left\|\mathcal{K}_{y} \varphi-\left(\varphi-\mathcal{N}\left(P_{\lambda}\left|u_{y}+\nu\right|^{p-2} \varphi\right)\right)\right\| \rightarrow 0 .
$$

Proof By the definition of $\mathcal{K}_{y}$, one has

$$
\begin{aligned}
\mathcal{K}_{y} \varphi-\left(\varphi-\mathcal{N}_{\lambda}\left(P_{\lambda}\left|u_{y}+v\right|^{p-2} \varphi\right)\right)= & \mathcal{N}_{\lambda}\left(\left|u_{y}+v\right|^{p-2} \varphi\right)-\sum_{j=1}^{n} \mathcal{N}\left(z^{p-2}\left(\cdot-y_{i}\right) \varphi\right) \\
& -\lambda \mathcal{N}_{\lambda}\left(b(x)\left|u_{y}+v\right|^{p-2} \varphi\right)+\sum_{i=1}^{n} L_{i} \varphi
\end{aligned}
$$

Obviously, $\mathcal{N}_{\lambda} \rightarrow \mathcal{N}$ in $\mathcal{L}\left(L^{\frac{p}{p-1}}\left(\mathbb{R}^{N}\right), H^{1}\left(\mathbb{R}^{N}\right)\right)$ as $\lambda \rightarrow 0$. Therefore, if $\lambda \rightarrow 0, v \in H^{1}\left(\mathbb{R}^{N}\right)$ with $v \rightarrow 0$, and $h \rightarrow \infty$, then for $\psi, \varphi \in H^{1}\left(\mathbb{R}^{N}\right)$, and uniformly in $y \in \mathcal{D}_{h}$,

$$
\begin{aligned}
& \left|\left\langle\mathcal{N}_{\lambda}\left(\left|u_{y}+v\right|^{p-2} \varphi\right)-\sum_{j=1}^{n} \mathcal{N}\left(z^{p-2}\left(\cdot-y_{i}\right) \varphi\right), \psi\right\rangle\right| \\
& \quad=\left|\left\langle\left(\mathcal{N}_{\lambda}-\mathcal{N}\right)\left(\left|u_{y}+v\right|^{p-2} \varphi\right), \psi\right\rangle\right|+\left|\left\langle\mathcal{N}\left(\left(\left|u_{y}+v\right|^{p-2}-\left|u_{y}\right|^{p-2}\right) \varphi\right), \psi\right\rangle\right|
\end{aligned}
$$




$$
\begin{aligned}
& \quad+\left|\left\langle\mathcal{N}\left(\left(\left|u_{y}\right|^{p-2}-\sum_{j=1}^{n} z^{p-2}\left(\cdot-y_{j}\right)\right) \varphi\right), \psi\right\rangle\right| \\
& \leq\left\|\left(\mathcal{N}_{\lambda}-\mathcal{N}\right)\left(\left|u_{y}+v\right|^{p-2} \varphi\right)\right\|\|\psi\|+C\left|\left(\left|u_{y}+v\right|^{p-2}-\left|u_{y}\right|^{p-2}\right)\right|_{L^{\frac{p}{p-2}\left(\mathbb{R}^{N}\right)}}\|\varphi\|\|\psi\| \\
& \quad+C\left|\left(\left|u_{y}\right|^{p-2}-\sum_{j=1}^{n} z^{p-2}\left(\cdot-y_{j}\right)\right)\right|_{L^{p-2}\left(\mathbb{R}^{N}\right)}\|\varphi\|\|\psi\| \\
& \rightarrow 0
\end{aligned}
$$

as a consequence of Lemmas 2.7 and 2.8. Moreover, by Lemma 2.6, for $\left|y_{i}-y_{j}\right| \rightarrow \infty(i \neq j)$, one sees that

$$
\sup _{y \in \mathcal{D}_{h}}\left\|\sum_{i=1}^{n} L_{i} \varphi\right\| \rightarrow 0 .
$$

For $\psi, \varphi \in H^{1}\left(\mathbb{R}^{N}\right)$

$$
\begin{aligned}
\lambda\left|\left\langle\mathcal{N}_{\lambda}\left(b(x)\left|u_{y}+v\right|^{p-2} \varphi\right), \psi\right\rangle\right|= & \lambda||\left\langle\left(\mathcal{N}_{\lambda}-\mathcal{N}\right)\left(b(x)\left|u_{y}+\nu\right|^{p-2} \varphi\right), \psi\right\rangle \mid \\
& +\left|\left\langle\mathcal{N}\left(b(x)\left|u_{y}+v\right|^{p-2} \varphi\right), \psi\right\rangle\right| \\
\leq & c \lambda\left\|\left(\mathcal{N}_{\lambda}-\mathcal{N}\right)\left(\left|u_{y}+\nu\right|^{p-2} \varphi\right)\right\|\|\psi\| \\
& +c \lambda\left\|u_{y}+v\right\|\|\varphi\|\|\psi\| \\
\rightarrow & 0
\end{aligned}
$$

as $\lambda \rightarrow 0$. We infer from (2.3)-(2.6) that, if $\lambda \rightarrow 0, v \in H^{1}\left(\mathbb{R}^{N}\right)$ with $v \rightarrow 0$, and $h \rightarrow \infty$,

$$
\sup _{y \in \mathcal{D}_{h}, \varphi \in H^{1}\left(\mathbb{R}^{N}\right),\|\varphi\|=1}\left\|\mathcal{K}_{y} \varphi-\left(\varphi-\mathcal{N}_{\lambda}\left(P_{\lambda}\left|u_{y}+\nu\right|^{p-2} \varphi\right)\right)\right\| \rightarrow 0
$$

Similar to above arguments, one can easily acquire the second conclusion of this lemma.

Clearly, the energy functional corresponding to the system $\left(\mathcal{S}_{\lambda}\right)$ is defined by

$$
\Phi_{\lambda}(u)=\frac{1}{2} \int_{\mathbb{R}^{N}}\left(|\nabla u|^{2}+V_{\lambda}|u|^{2}\right)-\frac{1}{p} \int_{\mathbb{R}^{N}} P_{\lambda}|u|^{p} \quad \text { for } u \in H^{1}\left(\mathbb{R}^{N}\right),
$$

where $V_{\lambda}=(1+\lambda a(x))$ and $P_{\lambda}=(1-\lambda b(x))$. It is easy to see that the critical points of $\Phi_{\lambda}$ are solutions of $\left(\mathcal{S}_{\lambda}\right)$. In the following, we shall use a Lyapunov-Schmidt reduction argument to find critical points of $\Phi_{\lambda}$. The first procedure is to convert the problem of finding critical points of $\Phi_{\lambda}$ to a finite dimensional problem, which consists of the following two lemmas.

Lemma 2.11 There exist $\lambda_{0}>0$ and $H_{0}>0$ such that, for $0<\lambda<\lambda_{0}$ and $h>H_{0}$, there exists a $C^{1}$-map

$$
v_{h, \lambda}: \mathcal{D}_{h} \rightarrow H^{1}\left(\mathbb{R}^{N}\right)
$$


(i) for any $y \in \mathcal{D}_{h}, v_{h, \lambda} \in \mathcal{W}_{y}$;

(ii) for any $y \in \mathcal{D}_{h}, \mathcal{P}_{y} \nabla \Phi_{\lambda}\left(u_{y}+v_{h, \lambda}\right)=0$, where $\mathcal{P}_{y}: H^{1}\left(\mathbb{R}^{N}\right) \rightarrow \mathcal{W}_{y}$ is the orthogonal projection onto $\mathcal{W}_{y}$;

(iii) $\lim _{\lambda \rightarrow 0, h \rightarrow \infty}\left\|v_{h, \lambda, y}\right\|=0$ uniformly in $y \in \mathcal{D}_{h} ; \lim _{|y| \rightarrow \infty}\left\|v_{h, \lambda, y}\right\|=0$ if $n=1$.

Decreasing $\lambda_{0}$ and increasing $H_{0}$ if necessary, we have the following result.

Lemma 2.12 For $0<\lambda<\lambda_{0}$ and $h>H_{0}$, if $y^{0}=\left(y_{1}^{0}, \ldots, y_{n}^{0}\right)$ is a critical point of $\Phi_{\lambda}\left(u_{y}+\right.$ $\left.v_{h, \lambda, y}\right)$, then $u_{y^{0}}+v_{h, \lambda, y^{0}}$ is a critical point of $\Phi_{\lambda}$.

Using Lemmas 2.9 and 2.10, repeating the arguments of Lemmas 2.6 and 2.7 in [40], one can easily prove Lemmas 2.11 and 2.12 .

\subsection{Estimates on $\Phi_{\lambda}\left(u_{y}+v_{h, \lambda, y}\right)$ and $v_{h, \lambda, y}$}

In order to prove Theorem 1.1 in the next section. We need first to estimate $\Phi_{\lambda}\left(u_{y}+v_{h, \lambda, y}\right)$ and $v_{h, \lambda, y}$. Denote $c_{0}=\Phi_{0}(z)$, where $\Phi_{0}$ is the functional $\Phi_{\lambda}$ with $\lambda=0$. Then

$$
c_{0}=\Phi_{0}(z)=\frac{1}{2} \int_{\mathbb{R}^{N}}\left(|\nabla z|^{2}+|z|^{2}\right)-\frac{1}{p} \int_{\mathbb{R}^{N}}|z|^{p} .
$$

In the following, we first estimate $\Phi_{\lambda}\left(u_{y}+v_{h, \lambda, y}\right)$. Note that

$$
\begin{aligned}
\Phi_{\lambda}\left(u_{y}+v_{h, \lambda, y}\right)= & \frac{1}{2} \int_{\mathbb{R}^{N}}\left|\nabla u_{y}+\nabla v_{h, \lambda, y}\right|^{2}+\frac{1}{2} \int_{\mathbb{R}^{N}}(1+\lambda a(x))\left|u_{y}+v_{h, \lambda, y}\right|^{2} \\
& -\frac{1}{p} \int_{\mathbb{R}^{N}}\left|u_{y}+v_{h, \lambda, y}\right|^{p}+\frac{\lambda}{p} \int_{\mathbb{R}^{N}} b(x)\left|u_{y}+v_{h, \lambda, y}\right|^{p} .
\end{aligned}
$$

A direct computation shows that

$$
\begin{aligned}
& \Phi_{\lambda}\left(u_{y}+v_{h, \lambda, y}\right) \\
& =\frac{1}{2} \sum_{i=1}^{n} \int_{\mathbb{R}^{N}}\left|\nabla z\left(x-y_{i}\right)\right|^{2}+\sum_{i=1}^{n} \int_{\mathbb{R}^{N}} \nabla z\left(x-y_{i}\right) \cdot \nabla\left(v_{h, \lambda, y}\right)+\frac{1}{2} \int_{\mathbb{R}^{N}}\left|\nabla\left(v_{h, \lambda, y}\right)\right|^{2} \\
& \quad+\sum_{i<j} \int_{\mathbb{R}^{N}} \nabla z\left(x-y_{i}\right) \cdot \nabla z\left(x-y_{j}\right)+\frac{1}{2} \sum_{i=1}^{n} \int_{\mathbb{R}^{N}}\left|z\left(x-y_{i}\right)\right|^{2}+\frac{1}{2} \int_{\mathbb{R}^{N}}\left|v_{h, \lambda, y}\right|^{2} \\
& \quad+\sum_{i=1}^{n} \int_{\mathbb{R}^{N}} z\left(x-y_{i}\right) \cdot v_{h, \lambda, y}+\sum_{i<j} \int_{\mathbb{R}^{N}} z\left(x-y_{i}\right) \cdot z\left(x-y_{j}\right) \\
& \quad+\frac{\lambda}{2} \int_{\mathbb{R}^{N}} a(x) u_{y}^{2}+\lambda \int_{\mathbb{R}^{N}} a(x) u_{y} v_{h, \lambda, y}+\frac{\lambda}{2} \int_{\mathbb{R}^{N}} a(x)\left(v_{h, \lambda, y}\right)^{2} \\
& \quad-\frac{1}{p} \int_{\mathbb{R}^{N}}\left|u_{y}+v_{h, \lambda, y}\right|^{p}+\frac{\lambda}{p} \int_{\mathbb{R}^{N}} b(x)\left|u_{y}+v_{h, \lambda, y}\right|^{p} .
\end{aligned}
$$

By Lemma 2.11, we may assume that $\left\|v_{h, \lambda, y}\right\| \leq 1$. Taking $a=u_{y}$ and $b=v_{h, \lambda, y}$ in Lemma 2.2, we have

$$
\frac{1}{p} \int_{\mathbb{R}^{N}}\left|u_{y}+v_{h, \lambda, y}\right|^{p}=\frac{1}{p} \int_{\mathbb{R}^{N}}\left|u_{y}\right|^{p}+\int_{\mathbb{R}^{N}}\left(u_{y}\right)^{p-1} v_{h, \lambda, y}+O\left(\left\|v_{h, \lambda, y}\right\|^{2}\right)
$$


and

$$
\frac{1}{p} \int_{\mathbb{R}^{N}} b(x)\left|u_{y}+v_{h, \lambda, y}\right|^{p}=\frac{1}{p} \int_{\mathbb{R}^{N}} b(x)\left|u_{y}\right|^{p}+\int_{\mathbb{R}^{N}} b(x)\left(u_{y}\right)^{p-1} v_{h, \lambda, y}+O\left(\left\|v_{h, \lambda, y}\right\|^{2}\right) .
$$

Here and in what follows, $O\left(\left\|v_{h, \lambda, y}\right\|^{2}\right)$ satisfies

$$
\left|O\left(\left\|v_{h, \lambda, y}\right\|^{2}\right)\right| \leq C\left\|v_{h, \lambda, y}\right\|^{2}
$$

for some positive constant $C$ independent of $h, \lambda, y$. Therefore, substituting (2.9) and (2.10) into (2.8), it follows that

$$
\begin{aligned}
\Phi_{\lambda}\left(u_{y}+v_{h, \lambda, y}\right) & \\
= & \frac{1}{2} \sum_{i=1}^{n} \int_{\mathbb{R}^{N}}\left|\nabla z\left(x-y_{i}\right)\right|^{2}+\sum_{i=1}^{n} \int_{\mathbb{R}^{N}} \nabla z\left(x-y_{i}\right) \cdot \nabla\left(v_{h, \lambda, y}\right)+\frac{1}{2} \int_{\mathbb{R}^{N}}\left|\nabla\left(v_{h, \lambda, y}\right)\right|^{2} \\
& +\sum_{i<j} \int_{\mathbb{R}^{N}} \nabla z\left(x-y_{i}\right) \cdot \nabla z\left(x-y_{j}\right)+\frac{1}{2} \sum_{i=1}^{n} \int_{\mathbb{R}^{N}}\left|z\left(x-y_{i}\right)\right|^{2}+\frac{1}{2} \int_{\mathbb{R}^{N}}\left|v_{h, \lambda, y}\right|^{2} \\
& +\sum_{i=1}^{n} \int_{\mathbb{R}^{N}} z\left(x-y_{i}\right) \cdot v_{h, \lambda, y}+\sum_{i<j} \int_{\mathbb{R}^{N}} z\left(x-y_{i}\right) \cdot z\left(x-y_{j}\right) \\
& +\frac{\lambda}{2} \int_{\mathbb{R}^{N}} a(x) u_{y}^{2}+\lambda \int_{\mathbb{R}^{N}} a(x) u_{y} v_{h, \lambda, y}+\frac{\lambda}{2} \int_{\mathbb{R}^{N}} a(x)\left(v_{h, \lambda, y}\right)^{2} \\
& -\frac{1}{p} \int_{\mathbb{R}^{N}}\left(u_{y}\right)^{p}-\int_{\mathbb{R}^{N}}\left(u_{y}\right)^{p-1} v_{h, \lambda, y}+\frac{\lambda}{p} \int_{\mathbb{R}^{N}} b(x)\left(u_{y}\right)^{p} \\
& +\int_{\mathbb{R}^{N}} b(x)\left(u_{y}\right)^{p-1} v_{h, \lambda, y}+O\left(\left\|v_{h, \lambda, y}\right\|^{2}\right) .
\end{aligned}
$$

Denote

$$
\begin{aligned}
\mathcal{K}_{y}= & -\sum_{i=1}^{n} \int_{\mathbb{R}^{N}} \nabla z\left(x-y_{i}\right) \cdot \nabla\left(v_{h, \lambda, y}\right)-\frac{1}{2} \int_{\mathbb{R}^{N}}\left|\nabla\left(v_{h, \lambda, y}\right)\right|^{2} \\
& -\sum_{i<j} \int_{\mathbb{R}^{N}} \nabla z\left(x-y_{i}\right) \cdot \nabla z\left(x-y_{j}\right) \\
& -\frac{1}{2} \int_{\mathbb{R}^{N}}\left(v_{h, \lambda, y}\right)^{2}-\sum_{i=1}^{n} \int_{\mathbb{R}^{N}} z\left(x-y_{i}\right) \cdot v_{h, \lambda, y}-\sum_{i<j} \int_{\mathbb{R}^{N}} z\left(x-y_{i}\right) \cdot z\left(x-y_{j}\right) \\
& -\lambda \int_{\mathbb{R}^{N}} a(x) u_{y} v_{h, \lambda, y}-\frac{\lambda}{2} \int_{\mathbb{R}^{N}} a(x)\left(v_{h, \lambda, y}\right)^{2}+\frac{1}{p} \int_{\mathbb{R}^{N}}\left(u_{y}\right)^{p}+\int_{\mathbb{R}^{N}}\left(u_{y}\right)^{p-1} v_{h, \lambda, y} \\
& -\lambda \int_{\mathbb{R}^{N}} b(x)\left(u_{y}\right)^{p-1} v_{h, \lambda, y}-\frac{1}{p} \sum_{i=1}^{n} \int_{\mathbb{R}^{N}} z^{p}\left(x-y_{i}\right)+O\left(\left\|v_{h, \lambda, y}\right\|^{2}\right) .
\end{aligned}
$$

Then

$$
\Phi_{\lambda}\left(u_{y}+v_{h, \lambda, y}\right)=n c_{0}+\frac{\lambda}{2} \int_{\mathbb{R}^{N}} a(x) u_{y}^{2}+\frac{\lambda}{p} \int_{\mathbb{R}^{N}} b(x) u_{y}^{p}-\mathcal{K}_{y}
$$


Thus, in order to estimate the functional $\Phi_{\lambda}\left(u_{y}+v_{h, \lambda, y}\right)$, it suffices to get the estimations for $\mathcal{K}_{y}$. Since

$$
\int_{\mathbb{R}^{N}} \nabla z\left(x-y_{i}\right) \cdot \nabla v+\int_{\mathbb{R}^{N}} z\left(x-y_{i}\right) v=\int_{\mathbb{R}^{N}} z^{p-1}\left(x-y_{i}\right) v, \quad \forall v \in H^{1}\left(\mathbb{R}^{N}\right),
$$

$\mathcal{K}_{y}$ can be rewritten as

$$
\begin{aligned}
\mathcal{K}_{y}= & -\sum_{i=1}^{n} \int_{\mathbb{R}^{N}} z^{p-1}\left(x-y_{i}\right) v_{h, \lambda, y}-\sum_{i<j} \int_{\mathbb{R}^{N}} z^{p-1}\left(x-y_{i}\right) z\left(x-y_{j}\right) \\
& -\lambda \int_{\mathbb{R}^{N}} a(x) u_{y} v_{h, \lambda, y}-\frac{\lambda}{2} \int_{\mathbb{R}^{N}} a(x)\left(v_{h, \lambda, y}\right)^{2}+\frac{1}{p} \int_{\mathbb{R}^{N}}\left(u_{y}\right)^{p}+\int_{\mathbb{R}^{N}}\left(u_{y}\right)^{p-1} v_{h, \lambda, y} \\
& -\lambda \int_{\mathbb{R}^{N}} b(x)\left(u_{y}\right)^{p-1} v_{h, \lambda, y}-\frac{1}{p} \sum_{i=1}^{n} \int_{\mathbb{R}^{N}} z^{p}\left(x-y_{i}\right)+O\left(\left\|v_{h, \lambda, y}\right\|^{2}\right)+\lambda O\left(\left\|v_{h, \lambda, y}\right\|^{2}\right) .
\end{aligned}
$$

Moreover, by the Hölder inequality one has

$$
\begin{aligned}
\lambda\left|\int_{\mathbb{R}^{N}} a(x) u_{y} v_{h, \lambda, y}\right| & \leq \lambda C\left(\int_{\mathbb{R}^{N}} a(x) u_{y}^{2}\right)^{\frac{1}{2}}\left\|v_{h, \lambda, y}\right\| \\
& \leq C \lambda^{2} \int_{\mathbb{R}^{N}} a(x) u_{y}^{2}+C\left\|v_{h, \lambda, y}\right\|^{2}
\end{aligned}
$$

and

$$
\begin{aligned}
\lambda\left|\int_{\mathbb{R}^{N}} b(x)\left(u_{y}\right)^{p-1} v_{h, \lambda, y}\right| & \leq C \lambda\left(\int_{\mathbb{R}^{N}} b(x) u_{y}^{p}\right)^{\frac{p-1}{p}}\left\|v_{h, \lambda, y}\right\| \\
& \leq C \lambda^{2} \int_{\mathbb{R}^{N}} b(x) u_{y}^{p}+C\left\|v_{h, \lambda, y}\right\|^{2} .
\end{aligned}
$$

Therefore, we have

$$
\begin{aligned}
\mathcal{K}_{y}= & \int_{\mathbb{R}^{N}}\left(u_{y}\right)^{p-1} v_{h, \lambda, y}-\sum_{i=1}^{n} \int_{\mathbb{R}^{N}} z^{p-1}\left(x-y_{i}\right) v_{h, \lambda, y}-\sum_{i<j} \int_{\mathbb{R}^{N}} z^{p-1}\left(x-y_{i}\right) z\left(x-y_{j}\right) \\
& +\frac{1}{p} \int_{\mathbb{R}^{N}}\left(u_{y}\right)^{p}-\frac{1}{p} \sum_{i=1}^{n} \int_{\mathbb{R}^{N}} z^{p}\left(x-y_{i}\right)+O\left(\left\|v_{h, \lambda, y}\right\|^{2}\right)+\lambda O\left(\left\|v_{h, \lambda, y}\right\|^{2}\right) \\
& +O\left(\lambda^{2}\left(\int_{\mathbb{R}^{N}} a(x) u_{y}^{2}+\int_{\mathbb{R}^{N}} b(x) u_{y}^{p}\right)\right) .
\end{aligned}
$$

Lemma 2.13 There exist $h_{0}>0, \lambda_{0}>0$, and $C_{i}>0(i=1,2,3)$ such that, if $0<\lambda \leq \lambda_{0}$, $h \geq h_{0}$, and $y \in \mathcal{D}_{h}$, then $\mathcal{K}_{y}$ satisfies

$$
\begin{aligned}
& \mathcal{K}_{y} \geq C \sum_{i<j} \int_{\mathbb{R}^{N}} z^{p-1}\left(x-y_{i}\right) z\left(x-y_{j}\right)-C_{1}\left\|v_{h, \lambda, y}\right\|^{2}-\lambda C_{2}\left\|v_{h, \lambda, y}\right\|^{2}-C_{3} \lambda^{2}, \\
& \mathcal{K}_{y} \leq C\left(\sum_{i<j} \int_{\mathbb{R}^{N}} z^{p-1}\left(x-y_{i}\right) z\left(x-y_{j}\right)+\left\|v_{h, \lambda, y}\right\|^{2}+\lambda\left\|v_{h, \lambda, y}\right\|^{2}-\lambda^{2}\right) .
\end{aligned}
$$


Proof From Lemmas 2.4 and 2.6, one sees that

$$
\begin{aligned}
& \left|\int_{\mathbb{R}^{N}}\left(u_{y}\right)^{p-1} v_{h, \lambda, y}-\sum_{i=1}^{n} \int_{\mathbb{R}^{N}} z^{p-1}\left(x-y_{i}\right) v_{h, \lambda, y}\right| \\
& \quad \leq\left(\int_{\mathbb{R}^{N}}\left(\left(u_{y}\right)^{p-1}-\sum_{i=1}^{n} z^{p-1}\left(x-y_{i}\right)\right)^{\frac{p}{p-1}}\right)^{\frac{p-1}{p}}\left(\int_{\mathbb{R}^{N}}\left|v_{h, \lambda, y}\right|^{p}\right)^{\frac{1}{p}} \\
& \quad \leq C\left(\int_{\mathbb{R}^{N}} \sum_{i \neq j} z^{p-1}\left(x-y_{i}\right) z\left(x-y_{j}\right)\right)^{\frac{p-1}{p}}\left\|v_{h, \lambda, y}\right\| \\
& \quad \leq C\left(\int_{\mathbb{R}^{N}} \sum_{i \neq j} z^{p-1}\left(x-y_{i}\right) z\left(x-y_{j}\right)\right)^{\frac{2(p-1)}{p}}+C\left\|v_{h, \lambda, y}\right\|^{2} \\
& \quad \leq C\left(\int_{\mathbb{R}^{N}} \sum_{i \neq j} z^{p-1}\left(x-y_{i}\right) z\left(x-y_{j}\right)\right)^{o(1)+C\left\|v_{h, \lambda, y}\right\|^{2} .}
\end{aligned}
$$

Moreover, by Lemma 2.3, we have

$$
\int_{\mathbb{R}^{N}} u_{y}^{p} \geq \sum_{i=1}^{n} \int_{\mathbb{R}^{N}} z^{p}\left(x-y_{i}\right)+2(p-1) \sum_{1 \leq i<j \leq n} \int_{\mathbb{R}^{N}} z^{p-1}\left(x-y_{i}\right) z\left(x-y_{j}\right)
$$

and by Lemma 2.1, one has

$$
\int_{\mathbb{R}^{N}} u_{y}^{p} \leq \sum_{i=1}^{n} \int_{\mathbb{R}^{N}} z^{p}\left(x-y_{i}\right)+C \sum_{1 \leq i<j \leq n} \int_{\mathbb{R}^{N}} z^{p-1}\left(x-y_{i}\right) z\left(x-y_{j}\right) .
$$

Here the fact

$$
\int_{\mathbb{R}^{N}} z^{p-1}\left(x-y_{i}\right) z\left(x-y_{j}\right)=\int_{\mathbb{R}^{N}} z\left(x-y_{i}\right) z^{p-1}\left(x-y_{j}\right)
$$

has been used. Substituting (2.13)-(2.15) into (2.12), one can easily get the desired conclusion.

Next, we are in a position to estimate $\left\|v_{h, \lambda, y}\right\|$.

Lemma 2.14 $\left\|v_{h, \lambda, y}\right\|$ satisfies

$$
\begin{aligned}
\left\|v_{h, \lambda, y}\right\| \leq & C \lambda\left(\int_{\mathbb{R}^{N}} a(x) u_{y}^{2}\right)^{\frac{1}{2}}+C \lambda\left(\int_{\mathbb{R}^{N}} b(x) u_{y}^{p}\right)^{\frac{p-1}{p}} \\
& +C\left(\sum_{i<j} \int_{\mathbb{R}^{N}} z^{p-1}\left(x-y_{i}\right) z\left(x-y_{j}\right)\right)^{\frac{p-1}{p}} .
\end{aligned}
$$

Proof By Lemma 2.11, for $v \in \mathcal{W}_{y}$, one has

$$
\begin{aligned}
0 & =\left\langle\nabla \Phi_{\lambda}\left(u_{y}+v_{h, \lambda, y}\right), v\right\rangle \\
& =\sum_{i=1}^{n} \int_{\mathbb{R}^{N}} \nabla z\left(x-y_{i}\right) \cdot \nabla v+\int_{\mathbb{R}^{N}} \nabla\left(v_{h, \lambda, y}\right) \cdot \nabla v
\end{aligned}
$$




$$
\begin{aligned}
& +\sum_{i=1}^{n} \int_{\mathbb{R}^{N}} z\left(x-y_{i}\right) v+\int_{\mathbb{R}^{N}} v_{h, \lambda, y} \nu+\lambda \sum_{i=1}^{n} \int_{\mathbb{R}^{N}} a(x) z\left(x-y_{i}\right) v \\
& +\lambda \int_{\mathbb{R}^{N}} a(x) v_{h, \lambda, y} v-\int_{\mathbb{R}^{N}} P_{\lambda}\left|u_{y}+v_{h, \lambda, y}\right|^{p-2}\left(u_{y}+v_{h, \lambda, y}\right) v .
\end{aligned}
$$

There exists $\theta \in(0,1)$ such that

$$
\begin{aligned}
& \int_{\mathbb{R}^{N}} P_{\lambda}\left|u_{y}+v_{h, \lambda, y}\right|^{p-2}\left(u_{y}+v_{h, \lambda, y}\right) v \\
& \quad=(p-1) \int_{\mathbb{R}^{N}} P_{\lambda}\left|u_{y}+\theta v_{h, \lambda, y}\right|^{p-2} v_{h, \lambda, y} \nu+\int_{\mathbb{R}^{N}} P_{\lambda} u_{y}^{p-1} \nu .
\end{aligned}
$$

Substituting (2.17) into (2.16) yields

$$
\begin{aligned}
\int_{\mathbb{R}^{N}} \nabla\left(v_{h, \lambda, y}\right) \cdot \nabla v+\int_{\mathbb{R}^{N}} v_{h, \lambda, y} v-(p-1) \int_{\mathbb{R}^{N}} P_{\lambda}\left|u_{y}+\theta v_{h, \lambda, y}\right|^{p-2} v_{h, \lambda, y} v \\
=-\lambda \int_{\mathbb{R}^{N}} a(x) v_{h, \lambda, y} v-\lambda \sum_{i=1}^{n} \int_{\mathbb{R}^{N}} a(x) z\left(x-y_{i}\right) v \\
\quad+\int_{\mathbb{R}^{N}} P_{\lambda} u_{y}^{p-1} v-\sum_{i=1}^{n} \int_{\mathbb{R}^{N}} z^{p-1}\left(x-y_{i}\right) v .
\end{aligned}
$$

Using the operator $\mathcal{N}$ and $\mathcal{P}_{y}$ defined in Section 2.1, we have

$$
\begin{aligned}
\left\langle v_{h, \lambda, y}-\right. & \left.\mathcal{P}_{y} \mathcal{N}\left(P_{\lambda}\left|u_{y}+\theta v_{h, \lambda, y}\right|^{p-2} v_{h, \lambda, y}\right), v\right\rangle \\
= & -\lambda \int_{\mathbb{R}^{N}} a(x) v_{h, \lambda, y} v-\lambda \sum_{i=1}^{n} \int_{\mathbb{R}^{N}} a(x) z\left(x-y_{i}\right) v \\
& +\int_{\mathbb{R}^{N}} P_{\lambda} u_{y}^{p-1} v-\sum_{i=1}^{n} \int_{\mathbb{R}^{N}} z^{p-1}\left(x-y_{i}\right) v .
\end{aligned}
$$

By Lemma 2.4, one has

$$
\begin{aligned}
& \left|\int_{\mathbb{R}^{N}} P_{\lambda} u_{y}^{p-1} v-\sum_{i=1}^{n} \int_{\mathbb{R}^{N}} z^{p-1}\left(x-y_{i}\right) v\right| \\
& \quad \leq\left(\int_{\mathbb{R}^{N}}\left|u_{y}^{p-1}-\sum_{i=1}^{n} z^{p-1}\left(x-y_{i}\right)\right| v \mid\right)+\lambda \int_{\mathbb{R}^{N}} b u_{y}^{p-1}|v| \\
& \quad \leq C\left(\int_{\mathbb{R}^{N}} \sum_{i \neq j} z^{p-1}\left(x-y_{i}\right) z\left(x-y_{j}\right)\right)^{\frac{p-1}{p}}\|v\|+\lambda C\left(\int_{\mathbb{R}^{N}} b u_{y}^{p}\right)^{\frac{p-1}{p}}\|v\| .
\end{aligned}
$$

Therefore, choosing $v=v_{h, \lambda, y}-\mathcal{P}_{y} \mathcal{N}\left(P_{\lambda}\left|u_{y}+\theta v_{h, \lambda, y}\right|^{p-2} v_{h, \lambda, y}\right) \in \mathcal{W}_{y}$ in (2.18) and using Lemmas 2.9 and 2.10, we obtain, for some $\eta>0$,

$$
\begin{aligned}
\eta\left\|v_{h, \lambda, y}\right\|\|v\| \leq & \lambda \int_{\mathbb{R}^{N}} a(x)\left|v_{h, \lambda, y} v\right|-\lambda \sum_{i=1}^{n} \int_{\mathbb{R}^{N}} a(x) z\left(x-y_{i}\right)|v| \\
& +C\left(\int_{\mathbb{R}^{N}} \sum_{i \neq j} z^{p-1}\left(x-y_{i}\right) z\left(x-y_{j}\right)\right)^{\frac{p-1}{p}}\|v\|+\lambda C\left(\int_{\mathbb{R}^{N}} b u_{y}^{p}\right)^{\frac{p-1}{p}}\|v\|,
\end{aligned}
$$


which implies, for $\lambda>0$ sufficiently small,

$$
\begin{aligned}
\left\|v_{h, \lambda, y}\right\|\|v\| \leq & C \lambda\left(\int_{\mathbb{R}^{N}} a(x) u_{y}^{2}\right)^{\frac{1}{2}}\|v\|+\lambda C\left(\int_{\mathbb{R}^{N}} b u_{y}^{p}\right)^{\frac{p-1}{p}}\|v\| \\
& +C\left(\int_{\mathbb{R}^{N}} \sum_{i \neq j} z^{p-1}\left(x-y_{i}\right) z\left(x-y_{j}\right)\right)^{\frac{p-1}{p}}\|v\| .
\end{aligned}
$$

Thus, we obtain the result.

\section{Proof of Theorem 1.1}

The main purpose of this section is to prove Theorem 1.1. For this, we shall prove that, for $\lambda>0$ small enough, we can choose $\mu(\lambda)$ large enough such that the function $\Phi_{\lambda}\left(u_{y}+v_{h, \lambda, y}\right)$ defined in Section 2.1 reaches its maximum in $\mathcal{D}_{\mu}$ at some point $y^{0}=\left(y_{1}^{0}, \ldots, y_{n}^{0}\right)$. Then $u_{y^{0}}+v_{h, \lambda, y^{0}}$ is a solution of $\left(\mathcal{S}_{\lambda}\right)$ by Lemma 2.12 .

We shall mainly consider the case $n \geq 2$ since the case $n=1$ is much easier. Define

$$
\gamma=\sup _{y \in\left(\mathbb{R}^{N}\right)^{n}}\left(\int_{\mathbb{R}^{N}} b(x) u_{y}^{p}(x)+\int_{\mathbb{R}^{N}} a(x) u_{y}^{2}(x)\right)
$$

By Lemmas 2.1 and 2.2, there exist $\lambda_{0}^{\prime}>0, h_{0}^{\prime}>$, and $C_{i}^{\prime}>0(i=1,2,3)$ such that, if $0<\lambda \leq$ $\lambda_{0}^{\prime}, h \geq h_{0}^{\prime}$, and $y \in \mathcal{D}_{h}$, then $\mathcal{K}_{y}$ satisfies

$$
\mathcal{K}_{y} \geq C_{1}^{\prime} \sum_{i<j} \int_{\mathbb{R}^{N}} z^{p-1}\left(x-y_{i}\right) z\left(x-y_{j}\right)-C_{2}^{\prime} \lambda^{2}-C_{3}^{\prime} \lambda^{3}
$$

Here and in the sequel, $C_{i}, C_{i}^{\prime}$, and $C$ are various positive constants independent of $\lambda$. We choose a number $k$ such that $k>\max \left\{1,12 \gamma / C_{1}^{\prime}\right\}$. Then, for any $\lambda$ satisfying

$$
0<\lambda<\lambda^{\prime}=\min \left\{\frac{\|z\|_{L^{p}}}{k}, \frac{k C_{1}^{\prime}}{2 C_{2}^{\prime}}, \sqrt{\frac{k C_{1}^{\prime}}{4 C_{3}^{\prime}}}, \lambda_{0}\right\},
$$

there exists $\mu^{*}=\mu^{*}(\lambda)>\mu=\mu(\lambda)>0$ such that, for $w \in \mathbb{R}^{N}$ with $|w| \in\left[\mu^{*}, \mu\right]$,

$$
k \lambda \leq \int_{\mathbb{R}^{N}} z^{p-1}(x) z(x-w) \leq 2 k \lambda
$$

Set

$$
\Gamma_{\lambda}=\left\{\Phi_{\lambda}\left(u_{y}+v_{h, \lambda, y}\right) \mid y \in \mathcal{D}_{\mu}\right\}
$$

To obtain an $n$-bump solution of $\left(\mathcal{S}_{\lambda}\right)$, it suffices to prove that $\Gamma_{\lambda}$ is achieved in the interior of $\mathcal{D}_{\mu}$.

Lemma 3.1 Assume $n \geq 2$. Then there exists $\lambda_{1} \in\left(0, \lambda^{\prime}\right)$ such that, for $0<\lambda<\lambda_{1}$,

$$
\Gamma_{\lambda}>\sup \left\{\Phi_{\lambda}\left(u_{y}+v_{h, \lambda, y}\right) \mid y \in \mathcal{D}_{\mu} \text { and }\left|y_{i}-y_{j}\right| \in\left[\mu^{*}, \mu\right] \text { for some } i \neq j\right\} \text {. }
$$


Proof Note that $\mu(\lambda) \rightarrow \infty$ as $\lambda \rightarrow 0$. By Lemma 2.2 and (3.3) we see that, if $y \in \mathcal{D}_{\mu(\lambda)}$, then

$$
\left\|v_{\mu, \lambda, y}\right\| \leq C \lambda^{\frac{p-1}{p}}
$$

Suppose that $y=\left(y_{1}, \ldots, y_{n}\right) \in \mathcal{D}_{\mu(\lambda)}$ and $\left|y_{i}-y_{j}\right| \in\left[\mu(\lambda), \mu^{*}(\lambda)\right]$ for some $i \neq j$. By (3.1)-(3.3), one has

$$
\mathcal{K}_{y} \geq C_{1}^{\prime} k \lambda-C_{2}^{\prime} \lambda^{2}-C_{3}^{\prime} \lambda^{3} \geq \frac{1}{2} C_{1}^{\prime} k \lambda-C_{3}^{\prime} \lambda^{3} \geq \frac{1}{4} C_{1}^{\prime} k \lambda \geq 3 \gamma \lambda .
$$

By (2.11) and (3.5), for $\lambda>0$ small enough, we obtain

$$
\begin{aligned}
\Phi_{\lambda}\left(u_{y}+v_{\mu, \lambda, y}\right) & =n c_{0}+\frac{\lambda}{2} \int_{\mathbb{R}^{N}} a(x) u_{y}^{2}+\frac{\lambda}{p} \int_{\mathbb{R}^{N}} b(x) u_{y}^{p}-\mathcal{K}_{y} \\
& \leq n c_{0}+\gamma \lambda-3 \gamma \lambda=n c_{0}-2 \gamma \lambda
\end{aligned}
$$

for $y=\left(y_{1}, \ldots, y_{n}\right) \in \mathcal{D}_{\mu(\lambda)}$ with $\left|y_{i}-y_{j}\right| \in\left[\mu(\lambda), \mu^{*}(\lambda)\right]$ for some $i \neq j$. On the other hand, if $y=\left(y_{1}, \ldots, y_{n}\right) \in \mathcal{D}_{\mu(\lambda)}$ and $\left|y_{i}-y_{j}\right| \rightarrow \infty$ for some $i \neq j$, then by (2.11) and Lemmas 2.1 and 2.2 , we have

$$
\begin{aligned}
\Phi_{\lambda}\left(u_{y}+v_{\mu, \lambda, y}\right)= & n c_{0}+\frac{\lambda}{2} \int_{\mathbb{R}^{N}} a(x) u_{y}^{2}+\frac{\lambda}{p} \int_{\mathbb{R}^{N}} b(x) u_{y}^{p}-\mathcal{K}_{y} \\
\geq & n c_{0}+\frac{\lambda}{p}\left(\int_{\mathbb{R}^{N}} a(x) u_{y}^{2}+\int_{\mathbb{R}^{N}} b(x) u_{y}^{p}\right)-C \lambda^{2} \\
& -C_{4} \lambda\left\|v_{\mu, \lambda, y}\right\|^{2}-C_{5}\left\|v_{\mu, \lambda, y}\right\|^{2}+o(1) \\
\geq & n c_{0}+\frac{\lambda}{p}\left(\int_{\mathbb{R}^{N}} a(x) u_{y}^{2}+\int_{\mathbb{R}^{N}} b(x) u_{y}^{p}\right)-C \lambda^{2} \\
& -C_{4}^{\prime}\left\|v_{\mu, \lambda, y}\right\|^{2}+o(1) \\
\geq & n c_{0}+\frac{\lambda}{p}\left(\int_{\mathbb{R}^{N}} a(x) u_{y}^{2}+\int_{\mathbb{R}^{N}} b(x) u_{y}^{p}\right)-C \lambda^{2} \\
& -\lambda^{2} C_{5}^{\prime}\left(\int_{\mathbb{R}^{N}} a(x) u_{y}^{2}+\int_{\mathbb{R}^{N}} b(x) u_{y}^{p}\right)+o(1),
\end{aligned}
$$

where $o(1)$ means some quantities which depend only on $y$ and converge to 0 as $\left|y_{i}-y_{j}\right| \rightarrow$ $\infty$ for all $i \neq j$. Therefore, for $\lambda$ small enough,

$$
\liminf _{\left|y_{i}-y_{j}\right| \rightarrow \infty, \forall i \neq j} \Phi_{\lambda}\left(u_{y}+v_{h, \lambda, y}\right) \geq n c_{0} .
$$

This inequality contradicts (3.6). Thus, we obtain the result.

We choose $y^{k}(\lambda)=\left(y_{1}^{k}(\lambda), \ldots, y_{n}^{k}(\lambda)\right) \in \mathcal{D}_{\mu(\lambda)}$ such that

$$
\lim _{k \rightarrow \infty} \Phi_{\lambda}\left(u_{y^{k}(\lambda)}+v_{\mu, \lambda, y^{k}(\lambda)}\right)=\Gamma_{\lambda}
$$

Then Lemma 3.1 implies that

$$
\inf _{k} \min _{i \neq j}\left|y_{i}^{k}(\lambda)-y_{j}^{k}(\lambda)\right| \geq \mu^{*}(\lambda)
$$


Therefore, for any $1 \leq i \leq n$, passing to a subsequence if necessary, we may assume either $\lim _{k \rightarrow \infty} y_{i}^{k}(\lambda)=y_{i}^{0}(\lambda)$ with $\left|y_{i}^{0}(\lambda)-y_{j}^{0}(\lambda)\right| \geq \mu^{*}$ for $i \neq j$ or $\lim _{k \rightarrow \infty} y_{i}^{k}(\lambda)=\infty$. Define

$$
\mathcal{U}(\lambda)=\left\{1 \leq i \leq n|| y_{i}^{k}(\lambda) \mid \rightarrow \infty, \text { as } k \rightarrow \infty\right\} .
$$

In the following, we shall prove that $\mathcal{U}(\lambda)=\emptyset$ for $\lambda>0$ sufficiently small and thus $\Phi_{\lambda}\left(u_{y}+\right.$ $\left.v_{h, \lambda, y}\right)$ attains its maximum at $\left(y_{1}^{0}(\lambda), \ldots, y_{n}^{0}(\lambda)\right) \in \mathcal{D}_{\mu(\lambda)}$.

Lemma 3.2 Assume $n \geq 2$. Then there exists $\lambda(n)>0$ such that for $\lambda \in(0, \lambda(n)), \mathcal{U}(\lambda)=\emptyset$.

Proof We adopt an argument borrowed from Lin and Liu [39, 40]. We argue by contradiction and assume that $\mathcal{U}(\lambda) \neq \emptyset$ along a sequence $\lambda_{m} \rightarrow 0$. Without loss of generality, we may assume $\mathcal{U}\left(\lambda_{m}\right)=\left\{1, \ldots, j_{n}\right\}$ for all $m \in \mathbb{N}$ and for some $1 \leq j_{n}<n$. The case in which $j_{n}=n$ can be handled similarly. For convenience of notations, we shall denote $\lambda_{m}=\lambda$, $y_{i}^{k}=y_{i}^{k}\left(\lambda_{m}\right), y^{k}=\left(y_{1}^{k}, \ldots, y_{n}^{k}\right), y_{*}^{k}=\left(y_{j_{n}+1}^{k}, \ldots, y_{n}^{k}\right)$, and $y_{*}^{0}=\left(y_{j_{n}+1}^{0}, \ldots, y_{n}^{0}\right)$ for $k=1,2, \ldots$ Then, as $k \rightarrow \infty$,

$$
\left|y_{1}^{k}\right| \rightarrow \infty, \quad \ldots, \quad\left|y_{j_{n}}^{k}\right| \rightarrow \infty
$$

and

$$
y_{j_{n}+1}^{k} \rightarrow y_{j_{n}+1}^{0}, \quad \ldots, \quad y_{n}^{k} \rightarrow y_{n}^{0} .
$$

Set

$$
w_{k}=\sum_{i=1}^{n} z\left(x-y_{i}^{k}\right), \quad w_{k, 1}=\sum_{i=1}^{j_{n}} z\left(x-y_{i}^{k}\right)
$$

and

$$
w_{k, 2}=\sum_{i=j_{n}+1}^{n} z\left(x-y_{i}^{k}\right), \quad w_{y_{*}^{0}}=\sum_{i=j_{n}+1}^{n} z\left(x-y_{i}^{0}\right) .
$$

Similar to (3.4), we have

$$
\left\|v_{\mu, \lambda, y^{k}}\right\| \leq C \lambda^{\frac{p-1}{p}}, \quad\left\|v_{\mu, \lambda, y_{*}^{k}}\right\| \leq C \lambda^{\frac{p-1}{p}}, \quad k=1,2, \ldots
$$

By (2.11), we obtain

$$
\begin{aligned}
\Phi_{\lambda}\left(w_{k}+v_{\mu, \lambda, y^{k}}\right)= & n c_{0}+\frac{\lambda}{2} \int_{\mathbb{R}^{N}} a(x) w_{k}^{2}+\frac{\lambda}{p} \int_{\mathbb{R}^{N}} b(x) w_{k}^{p}-\mathcal{K}_{y^{k}} \\
= & j_{n} c_{0}+\frac{\lambda}{2} \int_{\mathbb{R}^{N}} a(x) w_{k, 1}^{2}+\frac{\lambda}{2} \int_{\mathbb{R}^{N}} a(x) w_{k, 2}^{2}+\lambda \int_{\mathbb{R}^{N}} a(x) w_{k, 1} w_{k, 2} \\
& +\left(n-j_{n}\right) c_{0}+\frac{\lambda}{p} \int_{\mathbb{R}^{N}} b(x) w_{k}^{p}+\mathcal{K}_{y_{*}^{k}}-\mathcal{K}_{y^{k}}-\mathcal{K}_{y_{*}^{k}} \\
= & j_{n} c_{0}+\frac{\lambda}{2} \int_{\mathbb{R}^{N}} a(x) w_{k, 1}^{2}+\lambda \int_{\mathbb{R}^{N}} a(x) w_{k, 1} w_{k, 2}+\mathcal{K}_{y_{*}^{k}}-\mathcal{K}_{y^{k}} \\
& +\frac{\lambda}{p} \int_{\mathbb{R}^{N}} b(x)\left(w_{k}^{p}-w_{k, 2}^{p}\right)+\Phi_{\lambda}\left(w_{k, 2}+v_{\mu, \lambda, y_{*}^{k}}\right) .
\end{aligned}
$$


By Lemma 2.1, one sees

$$
\begin{aligned}
\int_{\mathbb{R}^{N}} b(x)\left(w_{k}^{p}-w_{k, 2}^{p}\right) \leq & C_{6} \sum_{i=1}^{j_{n}} \int_{\mathbb{R}^{N}} b(x) z^{p-1}\left(x-y_{i}^{k}\right) w_{k, 2} \\
& +C_{8} \sum_{i=1}^{j_{n}} \int_{\mathbb{R}^{N}} b(x) z^{p}\left(x-y_{j}^{k}\right) \\
& +C_{7} \sum_{j=j_{n}+1}^{n} \int_{\mathbb{R}^{N}} b(x) z^{p-1}\left(x-y_{j}^{k}\right) w_{k, 1} .
\end{aligned}
$$

Therefore, since $\left|y_{i}^{k}\right| \rightarrow \infty, i=1, \ldots, j_{n}$, as $k \rightarrow \infty$, we obtain

$$
\frac{\lambda}{2} \int_{\mathbb{R}^{N}} a(x) w_{k, 1}^{2}+\lambda \int_{\mathbb{R}^{N}} a(x) w_{k, 1} w_{k, 2} \rightarrow 0
$$

Furthermore, by (3.9) and the condition $\left(\mathcal{R}_{1}\right)$, we have

$$
\frac{\lambda}{p} \int_{\mathbb{R}^{N}} b(x)\left(w_{k}^{p}-w_{k, 2}^{p}\right) \rightarrow 0, \quad \text { as } k \rightarrow \infty
$$

From (3.8), (3.10) and (3.11), we arrive at

$$
\Phi_{\lambda}\left(w_{k}+v_{\mu, \lambda, y^{k}}\right) \leq \Phi_{\lambda}\left(w_{k, 2}+v_{\mu, \lambda, y_{*}^{k}}\right)+j_{n} c_{0}+\mathcal{K}_{y_{*}^{k}}-\mathcal{K}_{y^{k}}+o(1)
$$

Using Lemma 2.4, (3.3), and (3.7), we obtain

$$
\begin{aligned}
& \int_{\mathbb{R}^{N}}\left|\sum_{i=1}^{n} z^{p-1}\left(x-y_{i}^{k}\right)-\left(\sum_{i=1}^{n} z\left(x-y_{i}^{k}\right)\right)^{p-1}\right|\left|v_{\mu, \lambda, y^{k}}\right| \\
& \leq C\left(\sum_{i<j} \int_{\mathbb{R}^{N}} z^{p-1}\left(x-y_{i}^{k}\right) z\left(x-y_{j}^{k}\right)\right)^{\frac{p-1}{p}}\left\|v_{\mu, \lambda, y^{k}}\right\| \\
& \leq C^{\prime} \lambda^{\frac{2(p-1)}{p}} .
\end{aligned}
$$

From Lemma 2.2, (2.12), (3.7), and (3.13), one gets

$$
\begin{aligned}
\mathcal{K}_{y^{k}}= & \frac{1}{p} \int_{\mathbb{R}^{N}}\left(\sum_{i=1}^{n} z\left(x-y_{i}^{k}\right)\right)^{p}-\frac{1}{p} \sum_{i=1}^{n} \int_{\mathbb{R}^{N}} z^{p}\left(x-y_{i}^{k}\right) \\
& -\sum_{i<j} \int_{\mathbb{R}^{N}} z^{p-1}\left(x-y_{i}^{k}\right) z\left(x-y_{j}^{k}\right)+O\left(\lambda^{\frac{2(p-1)}{p}}\right) .
\end{aligned}
$$

In the same way, we have

$$
\begin{aligned}
\mathcal{K}_{y_{*}^{k}}= & \frac{1}{p} \int_{\mathbb{R}^{N}}\left(\sum_{i=j_{n}+1}^{n} z\left(x-y_{i}^{k}\right)\right)^{p}-\frac{1}{p} \sum_{i=j_{n}+1}^{n} \int_{\mathbb{R}^{N}} z^{p}\left(x-y_{i}^{k}\right) \\
& -\sum_{j_{n}<i<j} \int_{\mathbb{R}^{N}} z^{p-1}\left(x-y_{i}^{k}\right) z\left(x-y_{j}^{k}\right)+O\left(\lambda^{\frac{2(p-1)}{p}}\right) .
\end{aligned}
$$


We infer from (3.14) and (3.15) that

$$
\begin{aligned}
\mathcal{K}_{y_{*}^{k}}-\mathcal{K}_{y^{k}}= & \frac{1}{p} \int_{\mathbb{R}^{N}}\left(w_{k, 2}\right)^{p}-\frac{1}{p} \int_{\mathbb{R}^{N}}\left(w_{k}\right)^{p}+\frac{1}{p} \sum_{i=1}^{j_{n}} \int_{\mathbb{R}^{N}} z^{p}\left(x-y_{i}^{k}\right) \\
& +\sum_{i<j \leq j_{n}} \int_{\mathbb{R}^{N}} z^{p-1}\left(x-y_{i}^{k}\right) z\left(x-y_{j}^{k}\right)+O\left(\lambda^{\frac{2(p-1)}{p}}\right) \\
& +\sum_{i=1}^{j_{n}} \int_{\mathbb{R}^{N}} z^{p-1}\left(x-y_{i}^{k}\right) w_{k, 2} .
\end{aligned}
$$

By Lemma 2.3, the sum of the terms except $O\left(\lambda^{\frac{2(p-1)}{p}}\right)$ on the right side of (3.16) is negative. Thus, one has

$$
\mathcal{K}_{y_{*}^{k}}-\mathcal{K}_{y^{k}} \leq O\left(\lambda^{\frac{2(p-1)}{p}}\right)
$$

Letting $k \rightarrow \infty$, by (3.12), and using (3.17), we obtain

$$
\Gamma_{\lambda} \leq j_{n} c_{0}+\Phi_{\lambda}\left(w_{y_{*}^{0}}+v_{\mu, \lambda, y_{*}^{0}}\right)+C_{8}^{\prime} \lambda^{\frac{2(p-1)}{p}} .
$$

On the other hand, by Lemma 2.6 and (3.3), there exist $C_{9}, C_{10}>0$ such that

$$
C_{9} \lambda \leq \mu^{-\frac{N-2}{2}} e^{-\mu} \leq C_{10} \lambda
$$

which implies for $\lambda$ small enough

$$
(1-\delta) \ln \frac{1}{\lambda} \leq \mu=\mu(\lambda) \leq(1+\delta) \ln \frac{1}{\lambda}
$$

where $0<\delta<\frac{1}{p}$. We choose $\tau$ such that $0<\tau \leq \frac{p-2}{10 n p}$. By $\left(\mathcal{R}_{2}\right)$, there exists $R>0$ such that

$$
a(x) \geq e^{-\tau|x|}, \quad|x| \geq R
$$

or

$$
b(x) \geq e^{-\tau|x|}, \quad|x| \geq R .
$$

For $\lambda>0$ small enough, define

$$
\hat{y}_{s}^{\lambda}=\left(10 n \ln \frac{1}{\lambda}-4 s \mu(\lambda), 0, \ldots, 0\right) \in \mathbb{R}^{N}, \quad s=1,2, \ldots, n .
$$

The open balls $B\left(\hat{y}_{s}^{\lambda}, 2 \mu(\lambda)\right)$ are mutually disjoint. Thus there are $j_{n}$ integers from $\{1, \ldots, n\}$, denoted by $s_{1}<s_{2}<\cdots<s_{j_{n}}$, such that

$$
\left|\hat{y}_{s_{i}}^{\lambda}-y_{j}^{0}\right| \geq 2 \mu(\lambda), \quad i=1, \ldots, j_{n}, j=j_{n}+1, \ldots, n .
$$


Fang and Wang Boundary Value Problems (2015) 2015:9

Page 18 of 21

Denote $\hat{y}_{s_{i}}^{\lambda}$ by $y_{i}^{\lambda}$ for simplicity, $i=1, \ldots, j_{n}$. By (3.20), (3.23), and (3.24), one has

$$
\begin{aligned}
& R+1 \leq\left|y_{i}^{\lambda}\right| \leq 10 n \ln \frac{1}{\lambda}, \quad i=1, \ldots, j_{n}, \\
& \left|y_{i}^{\lambda}-y_{j}^{\lambda}\right| \geq 2 \mu(\lambda), \quad 1 \leq i<j \leq j_{n}, \\
& \left|y_{i}^{\lambda}-y_{j}^{0}\right| \geq 2 \mu(\lambda), \quad i=1, \ldots, j_{n}, j=j_{n}+1, \ldots, n .
\end{aligned}
$$

Therefore,

$$
\left(y_{1}^{\lambda}, \ldots, y_{j_{n}}^{\lambda}, y_{j_{n}+1}^{0}, \ldots, y_{n}^{0}\right) \in \mathcal{D}_{\mu(\lambda)}
$$

Denote $y^{\lambda}=\left(y_{1}^{\lambda}, \ldots, y_{j_{n}}^{\lambda}, y_{j_{n}+1}^{0}, \ldots, y_{n}^{0}\right)$. Set $w_{\lambda, 1}=\sum_{i=1}^{j_{n}} z\left(x-y_{i}^{\lambda}\right), w_{y_{*}^{0}}=\sum_{i=j_{n}+1}^{n} z\left(x-y_{i}^{0}\right)$, and $w_{\lambda}=w_{\lambda, 1}+w_{y_{*}^{0}}$. Similar to (3.8), one has

$$
\begin{aligned}
\Phi_{\lambda}\left(w_{\lambda}+v_{\mu, \lambda, y^{\lambda}}\right)= & j_{n} c_{0}+\frac{\lambda}{2} \int_{\mathbb{R}^{N}} a(x) w_{\lambda, 1}^{2}+\lambda \int_{\mathbb{R}^{N}} a(x) w_{\lambda, 1} w_{y_{*}^{0}}+\mathcal{K}_{y_{*}^{\lambda}}-\mathcal{K}_{y^{\lambda}} \\
& +\frac{\lambda}{p} \int_{\mathbb{R}^{N}} b(x)\left(w_{\lambda}^{p}-w_{y_{*}^{0}}^{p}\right)+\Phi_{\lambda}\left(w_{y_{*}^{0}}+v_{\mu, \lambda, y_{*}^{0}}\right) .
\end{aligned}
$$

As in (3.16), we have

$$
\begin{aligned}
\mathcal{K}_{y_{*}^{\lambda}}-\mathcal{K}_{y^{\lambda}}= & \frac{1}{p} \int_{\mathbb{R}^{N}}\left(w_{y_{*}^{0}}\right)^{p}-\frac{1}{p} \int_{\mathbb{R}^{N}}\left(w_{\lambda}\right)^{p}+\frac{1}{p} \sum_{i=1}^{j_{n}} \int_{\mathbb{R}^{N}} z^{p}\left(x-y_{i}^{\lambda}\right) \\
& +\sum_{i<j \leq j_{n}} z^{p-1}\left(x-y_{i}^{\lambda}\right) z\left(x-y_{j}^{\lambda}\right)+O\left(\lambda^{\frac{2(p-1)}{p}}\right) \\
& +\sum_{i=1}^{j_{n}} \int_{\mathbb{R}^{N}} z^{p-1}\left(x-y_{i}^{k}\right) w_{y_{*}^{0}} \\
\geq & \frac{1}{p} \int_{\mathbb{R}^{N}}\left(w_{y_{*}^{0}}\right)^{p}+\frac{1}{p} \sum_{i=1}^{j_{n}} \int_{\mathbb{R}^{N}} z^{p}\left(x-y_{i}^{\lambda}\right) \\
& -\frac{1}{p} \int_{\mathbb{R}^{N}}\left(w_{\lambda}\right)^{p}+O\left(\lambda^{\frac{2(p-1)}{p}}\right) .
\end{aligned}
$$

Together with Lemma 2.1 this implies that

$$
\begin{aligned}
\mathcal{K}_{y_{*}^{\lambda}}-\mathcal{K}_{y^{\lambda}} \geq & -C \sum_{i=1}^{j_{n}} \int_{\mathbb{R}^{N}} z^{p-1}\left(x-y_{i}^{\lambda}\right) w_{y_{*}^{0}}-C \sum_{i=1}^{j_{n}} \int_{\mathbb{R}^{N}}\left(w_{y_{*}^{0}}\right)^{p-1} z\left(x-y_{i}^{\lambda}\right) \\
& -C \sum_{1 \leq i<j \leq j_{n}} \int_{\mathbb{R}^{N}} z^{p-1}\left(x-y_{i}^{\lambda}\right) z\left(x-y_{j}^{\lambda}\right)+O\left(\lambda^{\frac{2(p-1)}{p}}\right) .
\end{aligned}
$$

By Lemma 2.6, (3.20), and (3.26), one sees that

$$
\begin{aligned}
\int_{\mathbb{R}^{N}} z^{p-1}\left(x-y_{i}^{\lambda}\right) z\left(x-y_{j}^{\lambda}\right) & \leq C_{11} e^{-2 \mu(\lambda)} \leq C_{12} e^{-2(1-\delta) \ln \frac{1}{\lambda}} \\
& =C_{12} \lambda^{2(1-\delta)} \leq C_{13} \lambda^{\frac{2(p-1)}{p}}
\end{aligned}
$$


In view of (3.27), a similar argument shows that

$$
\sum_{i=1}^{j_{n}} \int_{\mathbb{R}^{N}} z^{p-1}\left(x-y_{i}^{\lambda}\right) w_{y_{*}^{0}}+\sum_{i=1}^{j_{n}} \int_{\mathbb{R}^{N}}\left(w_{y_{*}^{0}}\right)^{p} z\left(x-y_{i}^{\lambda}\right) \leq C_{14} \lambda^{\frac{2(p-1)}{p}} .
$$

Combining (3.29)-(3.31), we have

$$
\mathcal{K}_{y_{*}^{\lambda}}-\mathcal{K}_{y^{\lambda}} \geq-C_{15} \lambda^{\frac{2(p-1)}{p}}
$$

Together with (3.28), it follows that

$$
\begin{aligned}
& \Phi_{\lambda}\left(u_{y^{\lambda}}+v_{\mu, \lambda, y^{\lambda}}\right) \geq j_{n} c_{0}+\frac{\lambda}{2} \int_{\mathbb{R}^{N}} a(x) w_{\lambda, 1}^{2}+\lambda \int_{\mathbb{R}^{N}} a(x) w_{\lambda, 1} w_{y_{*}^{0}}-C_{15} \lambda^{\frac{2(p-1)}{p}} \\
&+\frac{\lambda}{p} \int_{\mathbb{R}^{N}} b(x)\left(w_{y^{\lambda}}^{p}-w_{y_{*}^{0}}^{p}\right)+\Phi_{\lambda}\left(w_{y_{*}^{0}}+v_{\mu, \lambda, y_{*}^{0}}\right) .
\end{aligned}
$$

We distinguish the following two cases to finish the proof of this lemma.

(i) If (3.21) holds, then by (3.25), we have, for $i=1, \ldots, j_{n}$,

$$
\begin{aligned}
\int_{\mathbb{R}^{N}} a(x) w_{\lambda, 1}^{2} & \geq \int_{\left|x-y_{i}^{\lambda}\right| \leq 1} a(x) z^{2}\left(x-y_{i}^{\lambda}\right) \geq \int_{\left|x-y_{i}^{\lambda}\right| \leq 1} e^{-\tau|x|} z^{2}\left(x-y_{i}^{\lambda}\right) \\
& \geq C_{16} e^{-\tau\left|y_{i}^{\lambda}\right|} \geq C_{16} e^{-10 n \tau \ln \frac{1}{\lambda}}=C_{16} \lambda^{10 n \tau} .
\end{aligned}
$$

Hence,

$$
\Phi_{\lambda}\left(u_{y^{\lambda}}+v_{\mu, \lambda, y^{\lambda}}\right) \geq j_{n} c_{0}+\Phi_{\lambda}\left(w_{y_{*}^{0}}+v_{\mu, \lambda, y_{*}^{0}}\right)+C_{16} \lambda^{10 n \tau+1}-C_{15} \lambda^{\frac{2(p-1)}{p}} .
$$

Since $10 n \tau+1<\frac{2(p-1)}{p}$, we obtain, for $\lambda$ small enough,

$$
\Phi_{\lambda}\left(u_{y^{\lambda}}+v_{\mu, \lambda, y^{\lambda}}\right) \geq j_{n} c_{0}+\Phi_{\lambda}\left(w_{y_{*}^{0}}+v_{\mu, \lambda, y_{*}^{0}}\right)+C_{16}^{\prime} \lambda^{10 n \tau+1},
$$

which contradicts (3.18).

(ii) Suppose that (3.22) holds. Similar to (3.32), one has

$$
\begin{aligned}
\int_{\mathbb{R}^{N}} b(x)\left(u_{y^{\lambda}}^{p}-u_{y_{*}^{0}}^{p}\right) & \geq \int_{\left|x-y_{1}^{\lambda}\right| \leq 1} b(x) z^{p}\left(x-y_{1}^{\lambda}\right) \geq \int_{\left|x-y_{1}^{\lambda}\right| \leq 1} e^{-\tau|x|} z^{p}\left(x-y_{1}^{\lambda}\right) \\
& \geq C_{17} e^{-\tau\left|y_{1}^{\lambda}\right|} \geq C_{17} e^{-10 n \tau \ln \frac{1}{\lambda}}=C_{17} \lambda^{10 n \tau} .
\end{aligned}
$$

Repeating the arguments of (i), we get, for $\lambda$ small enough,

$$
\Phi_{\lambda}\left(u_{y^{\lambda}}+v_{\mu, \lambda, y^{\lambda}}\right) \geq j_{n} c_{0}+\Phi_{\lambda}\left(w_{y_{*}^{0}}+v_{\mu, \lambda, y_{*}^{0}}\right)+C_{17}^{\prime} \lambda^{10 n \tau+1} .
$$

This contradicts (3.18).

From (i) and (ii), we know that there exists $\lambda(n)>0$ such that, if $0<\lambda<\lambda(n)$, then $\mathcal{U}(\lambda)=$ $\emptyset$ and $\Phi_{\lambda}\left(u_{y}+v_{h, \lambda, y}\right)$ reaches its maximum at some point $\left(y_{1}^{0}, \ldots, y_{n}^{0}\right) \in \mathcal{D}_{\mu(\lambda)}$.

Next, we shall prove Theorem 1.1. 
Proof of Theorem 1.1 For $n \geq 2$, according to Lemma 3.2, if $0<\lambda<\lambda(n)$, then $\Phi_{\lambda}\left(u_{y}+v_{h, \lambda, y}\right)$ reaches its maximum at some point $y^{0}=\left(y_{1}^{0}, \ldots, y_{n}^{0}\right) \in \mathcal{D}_{\mu(\lambda)}$. Then $u_{y^{0}}+v_{h, \lambda, y^{0}}$ is an $n$-bump solution of $\left(\mathcal{S}_{\lambda}\right)$. For $n=1$, as a consequence of Lemma 2.11 (iii), if $\lambda \in\left(0, \lambda_{0}\right]$, then

$$
\lim _{|y| \rightarrow \infty} \Phi_{\lambda}\left(u_{y}+v_{h, \lambda, y}\right)=\Phi_{0}(z)=c_{0}
$$

Since $\Phi_{\lambda}\left(u_{y}+v_{h, \lambda, y}\right)$ is defined on all $\mathbb{R}^{N}, \Phi_{\lambda}\left(u_{y}+v_{h, \lambda, y}\right)$ has a critical point $y^{0} \in \mathbb{R}^{N}$ and $u_{y^{0}}+$ $v_{h, \lambda, y^{0}}$ is a 1-bump solution of $\left(\mathcal{S}_{\lambda}\right)$. By an argument similar to those in [34, 35], one sees that $u_{y^{0}}+v_{h, \lambda, y^{0}}$ is a positive solution of $\left(\mathcal{S}_{\lambda}\right)$. Set $\lambda(1)=\lambda_{0}$ and $\lambda_{1}(n)=\min \{\lambda(1), \ldots, \lambda(n)\}$. If $0<\lambda<\lambda_{1}(n)$, then $\left(\mathcal{S}_{\lambda}\right)$ has at least $n$ nontrivial positive solutions.

\section{Competing interests}

The authors declare that they have no competing interests.

\section{Authors' contributions}

All authors contributed equally to the writing of this paper. All authors read and approved the final manuscript.

\section{Acknowledgements}

This work was supported by Natural Science Foundation of China (11201186, 11071038, 11171135), NSF of Jiangsu Province (BK2012282), Jiangsu University foundation grant (11JDG117), China Postdoctoral Science Foundation funded project (2012M511199, 2013T60499).

Received: 8 September 2014 Accepted: 19 December 2014 Published online: 16 January 2015

\section{References}

1. Ambrosetti, A, Malchiodi, A, Secchi, S: Multiplicity results for some nonlinear Schrödinger equations with potentials. Arch. Ration. Mech. Anal. 159(3), 253-271 (2001)

2. Besieris, I-M: Solitons in randomly inhomogeneous media. In: Nonlinear Electromagnetics, pp. 87-116. Academic Press, New York (1980)

3. Byeon, J, Oshita, Y: Existence of multi-bump standing waves with a critical frequency for nonlinear Schrödinger equations. Commun. Partial Differ. Equ. 29(11-12), 1877-1904 (2004)

4. Cingolani, S, Nolasco, M: Multi-peak periodic semiclassical states for a class of nonlinear Schrödinger equations. Proc. R. Soc. Edinb., Sect. A 128(6), 1249-1260 (1998)

5. Kang, X-S, Wei, J-C: On interacting bumps of semi-classical states of nonlinear Schrödinger equations. Adv. Differ. Equ. 5(7-9), 899-928 (2000)

6. Li, Y-Y: On a singularly perturbed elliptic equation. Adv. Differ. Equ. 2(6), 955-980 (1997)

7. Oh, Y-G: On positive multi-lump bound states of nonlinear Schrödinger equations under multiple well potential. Commun. Math. Phys. 131(2), 223-253 (1990)

8. Floer, A, Weinstein, A: Nonspreading wave packets for the cubic Schrödinger equation with a bounded potential. J. Funct. Anal. 69(3), 397-408 (1986)

9. Oh, Y-G: Existence of semiclassical bound states of nonlinear Schrödinger equations with potentials of the class $(V)_{a}$. Commun. Partial Differ. Equ. 13(12), 1499-1519 (1988)

10. Rabinowitz, P-H: On a class of nonlinear Schrödinger equations. Z. Angew. Math. Phys. 43(2), 270-291 (1992)

11. Del Pino, M, Felmer, P-L: Local mountain passes for semilinear elliptic problems in unbounded domains. Calc. Var. Partial Differ. Equ. 4(2), 121-137 (1996)

12. Del Pino, M, Felmer, P-L: Multi-peak bound states for nonlinear Schrödinger equations. Ann. Inst. Henri Poincaré, Anal. Non Linéaire 15(2), 127-149 (1998)

13. Ambrosetti, A, Badiale, M, Cingolani, S: Semiclassical states of nonlinear Schrödinger equations. Arch. Ration. Mech. Anal. 140(3), 285-300 (1997)

14. Del Pino, M, Felmer, P-L: Semi-classical states of nonlinear Schrödinger equations: a variational reduction method. Math. Ann. 324(1), 1-32 (2002)

15. Del Pino, M, Felmer, P-L: Semi-classical states for nonlinear Schrödinger equations. J. Funct. Anal. 149(1), 245-265 (1997)

16. Ambrosetti, A, Malchiodi, A: Perturbation Methods and Semilinear Elliptic Problems on $\mathbf{R}^{n}$. Progress in Mathematics, vol. 240. Birkhäuser, Basel (2006)

17. Ambrosetti, A, Malchiodi, A, Ni, W-M: Singularly perturbed elliptic equations with symmetry: existence of solutions concentrating on spheres. I. Commun. Math. Phys. 235(3), 427-466 (2003)

18. Del Pino, M, Kowalczyk, M, Wei, J-C: Concentration on curves for nonlinear Schrödinger equations. Commun. Pure Appl. Math. 60(1), 113-146 (2007)

19. Byeon, J, Wang, Z-Q: Standing waves with a critical frequency for nonlinear Schrödinger equations. Arch. Ration. Mech. Anal. 165(4), 295-316 (2002)

20. Byeon, J, Wang, Z-Q: Standing waves with a critical frequency for nonlinear Schrödinger equations. II. Calc. Var. Partial Differ. Equ. 18(2), 207-219 (2003) 
21. Cao, D-M, Noussair, E-S: Multi-bump standing waves with a critical frequency for nonlinear Schrödinger equations. J. Differ. Equ. 203(2), 292-312 (2004)

22. Cao, D-M, Peng, S-J: Multi-bump bound states of Schrödinger equations with a critical frequency. Math. Ann. 336(4), 925-948 (2006)

23. Ding, Y-H, Lin, F-H: Solutions of perturbed Schrödinger equations with critical nonlinearity. Calc. Var. Partial Differ. Equ. 30(2), 231-249 (2007)

24. Ding, Y-H, Szulkin, A: Bound states for semilinear Schrödinger equations with sign-changing potential. Calc. Var. Partial Differ. Equ. 29(3), 397-419 (2007)

25. Ding, Y-H, Wei, J-C: Semiclassical states for nonlinear Schrödinger equations with sign-changing potentials. J. Funct. Anal. 251(2), 546-572 (2007)

26. Coti Zelati, V, Rabinowitz, P-H: Homoclinic type solutions for a semilinear elliptic PDE on $\mathbf{R}^{n}$. Commun. Pure Appl. Math. 45(10), 1217-1269 (1992)

27. Coti Zelati, V, Rabinowitz, P-H: Homoclinic orbits for second order Hamiltonian systems possessing superquadratic potentials. J. Am. Math. Soc. 4(4), 693-727 (1991)

28. Alama, S, Li, YYY: On 'multibump' bound states for certain semilinear elliptic equations. Indiana Univ. Math. J. 41(4), 983-1026 (1992)

29. Cerami, G, Devillanova, G, Solimini, S: Infinitely many bound states for some nonlinear scalar field equations. Calc. Var. Partial Differ. Equ. 23(2), 139-168 (2005)

30. Cerami, G, Passaseo, D, Solimini, S: Infinitely many positive solutions to some scalar field equations with nonsymmetric coefficients. Commun. Pure Appl. Math. 66(3), 372-413 (2013)

31. Ackermann, $\mathrm{N}$, Weth, T: Multibump solutions of nonlinear periodic Schrödinger equations in a degenerate setting. Commun. Contemp. Math. 7(3), 269-298 (2005)

32. Ackermann, N: A nonlinear superposition principle and multibump solutions of periodic Schrödinger equations. J. Funct. Anal. 234(2), 277-320 (2006)

33. Rabinowitz, P-H: A multibump construction in a degenerate setting. Calc. Var. Partial Differ. Equ. 5(2), 159-182 (1997)

34. Liu, Z-L, Wang, Z-Q: Multi-bump type nodal solutions having a prescribed number of nodal domains. I. Ann. Inst. Henri Poincaré, Anal. Non Linéaire 22(5), 597-608 (2005)

35. Liu, Z-L, Wang, Z-Q: Multi-bump type nodal solutions having a prescribed number of nodal domains. II. Ann. Inst. Henri Poincaré, Anal. Non Linéaire 22(5), 609-631 (2005)

36. Lin, L-S, Liu, Z-L: Multi-bubble solutions for equations of Caffarelli-Kohn-Nirenberg type. Commun. Contemp. Math. 13(6), 945-968 (2011)

37. Wang, J, Xu, J-X, Zhang, F-B, Chen, X-M: Existence of multi-bump solutions for a semilinear Schrödinger-Poisson system. Nonlinearity 26(5), 1377-1399 (2013)

38. Pi, H-R, Wang, C-H: Multi-bump solutions for nonlinear Schrödinger equations with electromagnetic fields. ESAIM Control Optim. Calc. Var. 19(1), 91-111 (2013)

39. Lin, L-S, Liu, Z-L: Multi-bump solutions and multi-tower solutions for equations on $\mathbb{R}^{N}$. J. Funct. Anal. 257(2), 485-505 (2009)

40. Lin, L-S, Liu, Z-L, Chen, X-W: Multi-bump solutions for a semilinear Schrödinger equation. Indiana Univ. Math. J. 58(4), 1659-1689 (2009)

41. D'Aprile, T, Wei, J-C: Standing waves in the Maxwell-Schrödinger equation and an optimal configuration problem. Calc. Var. Partial Differ. Equ. 25(1), 105-137 (2006)

42. Ambrosetti, A, Badiale, M: Homoclinics: Poincaré-Melnikov type results via a variational approach. Ann. Inst. Henri Poincaré, Anal. Non Linéaire 15(2), 233-252 (1998)

43. Berestycki, H, Lions, P-L: Nonlinear scalar field equations. II. Existence of infinitely many solutions. Arch. Ration. Mech. Anal. 82(4), 347-375 (1983)

44. Kwong, M-K: Uniqueness of positive solutions of $\Delta u-u+u^{p}=0$ in $\mathbf{R}^{n}$. Arch. Ration. Mech. Anal. 105(3), 243-266 (1989)

45. Ni, W-M, Takagi, I: Locating the peaks of least-energy solutions to a semilinear Neumann problem. Duke Math. J. 70(2), 247-281 (1993)

46. Cerami, G, Passaseo, D: Existence and multiplicity results for semilinear elliptic Dirichlet problems in exterior domains Nonlinear Anal. 24(11), 1533-1547 (1995)

47. Bahri, A, Lions, P-L: On the existence of a positive solution of semilinear elliptic equations in unbounded domains. Ann. Inst. Henri Poincaré, Anal. Non Linéaire 14(3), 365-413 (1997)

\section{Submit your manuscript to a SpringerOpen ${ }^{\ominus}$ journal and benefit from:}

- Convenient online submission

Rigorous peer review

- Immediate publication on acceptance

- Open access: articles freely available online

- High visibility within the field

- Retaining the copyright to your article 